\title{
Sex differences in the manifestation and evolution of coronary artery plaques
}

\author{
Benedikt Bernhard ${ }^{1} \cdot$ Christoph Gräni ${ }^{1}$
}

Published online: 11 May 2021

(c) The Author(s), under exclusive licence to Springer Nature B.V. 2021

\section{Editorial}

Even though the past decades have brought an increase in the understanding of the pathophysiology of coronary artery disease (CAD), several aspects remain incompletely understood. This is especially true for sex-specific differences in the development, manifestation, and prognosis of coronary artery plaques. While the prevalence of obstructive CAD is higher in men compared to women, non-obstructive CAD and ischemia with no obstructive coronary arteries (INOCA) are relatively more common in women [1-3]. Compared to obstructive CAD, fewer established preventive, diagnostic and treatment options are available for INOCA [4], complicating treatment of CAD, especially in younger women at low cardiovascular risk. Atypical symptoms in the clinical setting of suspected CAD are more common in women than in men, which might lead to delayed detection of CAD and acute coronary syndrome [5]. Women present with the disease at higher age [6] and are at higher risk for major adverse cardiovascular events and fatal outcome of myocardial infarction compared to men in the presence of obstructive, and as well in non-obstructive CAD [5, 7-9]. Sexdependent disparities seem to diminish when women reach menopause and similar CAD prevalence rates and mortality rates in patients with myocardial infarction are observed in the seventh decade of life for men and women [8,9].

Several models were established to explain these findings and encompass sex-specific exposure to cardiovascular risk factors (CVRF), differences in the pathophysiology of plaque formation, and the protective impact of estrogen. Beyond traditional CVRF, several sex-specific riskenhancing conditions may be present in women: the higher prevalence of depression in women, pregnancy-associated

Christoph Gräni

christoph.graeni@insel.ch

1 Department of Cardiology, Inselspital, Bern University Hospital, University of Bern, 3010 Bern, Switzerland disorders like preeclampsia or gestational diabetes mellitus and arterial hypertension, delivery of a low-birth weight infant, pregnancy-associated cessation of statin intake, and premature menopause [10-12]. Loss of the ovarian function and the protective effect of estrogen after menopause may foster activation of the renin-angiotensin-aldosterone system, left ventricular remodeling, and endothelial dysfunction [13]. Furthermore, estrogen-mediated effects on the lipid- and cholesterol metabolism of the liver are proposed to modify the cardiovascular risk [14]. Although these findings implicate a cardioprotective effect of estrogen, hormone replacement therapy has failed to modulate the progression of CAD in postmenopausal patients $[15,16]$. The interaction of hormones, traditional CVRF, and risk-enhancing conditions seems to be more complex than assumed and further evidence on sex differences in the pathophysiology of CAD is warranted. In this regard, the formation of atherosclerotic plaques and the change of plaque morphology over time is crucial to understand the evolution from asymptomatic nonobstructive CAD to acute myocardial infarction.

Previous studies could identify different characteristics of coronary artery plaques in men and women. Due to the heterogeneity between studies in terms of imaging modalities (invasive optical coherence tomography (OCT) [17, 18] versus invasive intravascular ultrasound (IVUS) [19-21] versus noninvasive imaging (i.e. coronary computed tomography angiography; CCTA) [1, 22-24]) and investigated lesions (non-obstructive or obstructive [1, 18, 23, 24] versus culprit lesions in myocardial infarction $[17,19])$ results concerning sex-differences were incoherent. In patients undergoing CCTA, the following findings were consistent between most studies: Men have higher plaque burden in terms of number of plaques and plaque volume, obstructive disease is more common in men, and the relative number of non-calcified plaque is higher in women than in men [1, 22-24]. On contrary, OCT and IVUS findings suggest a higher prevalence of lipid-core plaques in men compared to women $[18,21]$. Due to the invasive nature of OCT and IVUS, these findings 
might be related to the higher number of patients included with acute coronary syndrome compared to CCTA studies where mostly patients at low- to intermediate pretest probability for CAD were assessed. Although there exists evidence on sex-differences of coronary plaques, most studies were limited to a one-time point assessment of the plaque burden and hence their results only represent a snapshot in the continuum of CAD. So far, little is known about sexdependent changes of coronary plaques over time in intermediate and low-risk patients.

In this context, we congratulate on the important presented study by El Mahdiui et al., which provides further insights into sex differences in the evolution of coronary plaques. The authors sought to analyze sex disparities in coronary plaque morphology changes by serial non-invasive imaging using CCTA in a low- to intermediate CAD risk population from the multinational, multicenter prospective SMARTool study (Simulation Modeling of coronary ARTery disease: a tool for clinical decision support).

In a total of 211 patients, recruited in seven centers of five European countries who underwent clinically indicated CCTA, after a mean time of about 6 years a second CCTA was conducted to assess changes in coronary plaque morphology. The main findings were that prevalence of coronary plaques and mean percentage fibro-fatty atheroma volume (PAV) were consistently higher in men than in women. In the subgroup analysis, where patients were dichotomized by age groups, a more pronounced regression of fibrous and non-calcified PAV in women under 55 years of age compared to age-matched men was revealed. No differences in the change of total or compositional PAV were observed between women and men of 55 years or older. The results implicate a possible association of plaque formation to estrogen levels, since regression of fibrous and non-calcified PAV was observed only in women expected to be premenopausal, but not in men or older women. Finally, the present study could confirm some general findings on sex disparities in CAD: women with CAD were older than men, had higher high-density lipoprotein (HDL) levels, and presented more often with atypical symptoms compared to men.

Strengths of the presented study are the multicentric design, the serial CCTA assessment over time, the interpretation of the CCTAs in a core laboratory and the long follow-up. Limitations are based on the fact that women were underrepresented (31\%) which complicates the statistical comparision to the larger group of men and the nature of the study, which did not link changes in plaque morphology to clinical outcomes. Plaque burden is an important outcome predictor and findings on sex-discrepancy would have helped to understand the evolution from stable plaque to myocardial infarction in men and women. The proposed associations of plaque changes to menopause should be interpreted with caution since it was made on the observation of differences between age-groups by a cut-off of 55 years. The presence of menopause is not an exclusively age-dependent variable and should have been determined by either standardized questionnaires or sex hormone levels, rather than stratified according to agegroups. Nevertheless, the finding is in line with previous studies, implicating a protective effect of estrogen. Sex differences in CAD remain a timely topic because the management of CAD in women is still suboptimal. Women with suspected CAD are less likely to be diagnosed early, receive less preventive care and are treated less adequately for CAD [6]. Patient education of women who are at high cardiovascular risk is worse compared to men and women are less aware of the fact that they are at high risk and hence present later at the emergency unit when they suffer from acute coronary syndrome [5, 25]. Modifiable risk factors explain $94 \%$ of the occurrence of myocardial infarction in women and preventive treatment is a powerful tool to improve survival [26]. Nevertheless, guidance on approaches to modify risk factors is often lacking in women [25]. The presented study showed sexdifferences in the development of coronary artery plaques and promotes the establishment of sex-based management of CAD. Encouragingly, in recent years, awareness for sex-specific factors of CAD is rising in risk factor management, and recently published guidelines include sexspecific CVRF in the decision process for statin therapy [27]. However, the therapeutic implications of changes in non-obstructive plaque morphology in men versus women remain to be elucidated. There are no guidelines or consensus that specifically address possible treatment adaption according to plaque morphology changes over time. Development of further sex-based management according to imaging-based findings should be pursued in the future to guarantee optimal tailoring of therapy for men and women with CAD.

\section{Declarations}

Conflict of interest The authors declare that they have no conflict of interest.

\section{References}

1. Plank F et al (2019) Sex differences in coronary artery plaque composition detected by coronary computed tomography: quantitative and qualitative analysis. Neth Heart J 27(5):272-280

2. Waheed $\mathrm{N}$ et al (2020) Sex differences in non-obstructive coronary artery disease. Cardiovasc Res 116(4):829-840

3. Safdar B et al (2018) Presentation, clinical profile, and prognosis of young patients with myocardial infarction with nonobstructive 
coronary arteries (MINOCA): results from the VIRGO Study. J Am Heart Assoc 7(13):e009174

4. Turgeon RD, Pearson GJ, Graham MM (2018) Pharmacologic treatment of patients with myocardial ischemia with no obstructive coronary artery disease. Am J Cardiol 121(7):888-895

5. Mehilli J, Presbitero P (2020) Coronary artery disease and acute coronary syndrome in women. Heart 106(7):487-492

6. Anand SS et al (2008) Risk factors for myocardial infarction in women and men: insights from the INTERHEART study. Eur Heart J 29(7):932-940

7. Sedlak TL et al (2013) Sex differences in clinical outcomes in patients with stable angina and no obstructive coronary artery disease. Am Heart J 166(1):38-44

8. Bairey Merz CN et al (2006) Insights from the NHLBI-Sponsored Women's Ischemia Syndrome Evaluation (WISE) Study: Part II: gender differences in presentation, diagnosis, and outcome with regard to gender-based pathophysiology of atherosclerosis and macrovascular and microvascular coronary disease. J Am Coll Cardiol 47(3 Suppl):S21-S29

9. Champney KP et al (2009) The joint contribution of sex, age and type of myocardial infarction on hospital mortality following acute myocardial infarction. Heart 95(11):895-899

10. Agarwala A et al (2020) The use of sex-specific factors in the assessment of women's cardiovascular risk. Circulation 141(7):592-599

11. Grandi SM et al (2017) Hypertensive disorders in pregnancy and the risk of subsequent cardiovascular disease. Paediatr Perinat Epidemiol 31(5):412-421

12. Muka $\mathrm{T}$ et al (2016) Association of age at onset of menopause and time since onset of menopause with cardiovascular outcomes, intermediate vascular traits, and all-cause mortality: a systematic review and meta-analysis. JAMA Cardiol 1(7):767-776

13. Zhao $\mathrm{Z}$ et al (2014) Role of estrogen in diastolic dysfunction. Am J Physiol Heart Circ Physiol 306(5):H628-H640

14. Palmisano BT, Zhu L, Stafford JM (2017) Role of estrogens in the regulation of liver lipid metabolism. Adv Exp Med Biol 1043:227-256

15. Herrington DM et al (2000) Effects of estrogen replacement on the progression of coronary-artery atherosclerosis. N Engl J Med 343(8):522-529

16. Hodis $\mathrm{HN}$ et al (2003) Hormone therapy and the progression of coronary-artery atherosclerosis in postmenopausal women. $\mathrm{N}$ Engl J Med 349(6):535-545

17. Sato $\mathrm{T}$ et al (2020) Age- and gender-related differences in coronary lesion plaque composition on optical coherence tomography. Circ J 84(3):463-470
18. Tian J et al (2019) Gender differences in plaque characteristics of nonculprit lesions in patients with coronary artery disease. BMC Cardiovasc Disord 19(1):45

19. Ann SH et al (2016) Gender differences in plaque characteristics of culprit lesions in patients with ST elevation myocardial infarction. Heart Vessels 31(11):1767-1775

20. Ruiz-García J et al (2012) Age- and gender-related changes in plaque composition in patients with acute coronary syndrome: the PROSPECT study. EuroIntervention 8(8):929-938

21. Inoue $\mathrm{F}$ et al (2010) Gender differences in coronary plaque characteristics in patients with stable angina: a virtual histology intravascular ultrasound study. Cardiovasc Interv Ther 25(1):40-45

22. Nasir K et al (2010) Noninvasive assessment of gender differences in coronary plaque composition with multidetector computed tomographic angiography. Am J Cardiol 105(4):453-458

23. Qureshi W et al (2013) Gender differences in coronary plaque composition and burden detected in symptomatic patients referred for coronary computed tomographic angiography. Int J Cardiovasc Imaging 29(2):463-469

24. Blaha MJ et al (2009) Gender differences in coronary plaque composition by coronary computed tomography angiography. Coron Artery Dis 20(8):506-512

25. Leifheit-Limson EC et al (2015) Sex differences in cardiac risk factors, perceived risk, and health care provider discussion of risk and risk modification among young patients with acute myocardial infarction: the VIRGO Study. J Am Coll Cardiol 66(18):1949-1957

26. Yusuf S et al (2004) Effect of potentially modifiable risk factors associated with myocardial infarction in 52 countries (the INTERHEART study): case-control study. The Lancet 364(9438):937-952

27. Grundy SM et al (2019) 2018 AHA/ACC/AACVPR/AAPA/ABC/ ACPM/ADA/AGS/APhA/ASPC/NLA/PCNA Guideline on the Management of Blood Cholesterol: a report of the American College of Cardiology/American Heart Association Task Force on Clinical Practice Guidelines. Circulation 139(25):e1082-e1143

Publisher's Note Springer Nature remains neutral with regard to jurisdictional claims in published maps and institutional affiliations. 